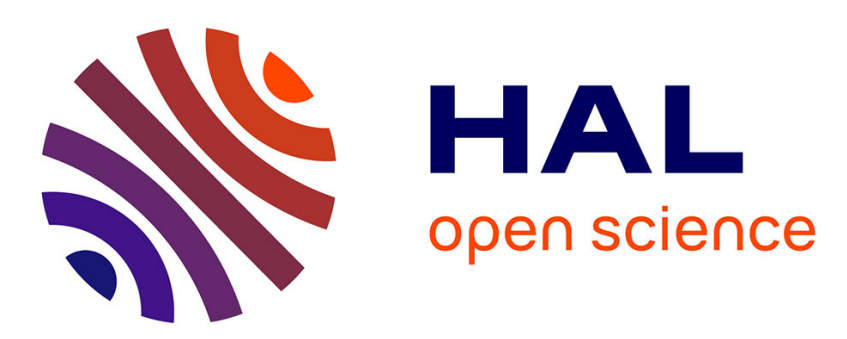

\title{
Possibilités d'application des couches minces magnétiques à la détection des champs magnétiques faibles \\ B. Waksmann
}

\section{- To cite this version:}

B. Waksmann. Possibilités d'application des couches minces magnétiques à la détection des champs magnétiques faibles. Revue de Physique Appliquée, 1970, 5 (1), pp.145-149. 10.1051/rphysap:0197000501014500 . jpa-00243349

HAL Id: jpa-00243349

https://hal.science/jpa-00243349

Submitted on 1 Jan 1970

HAL is a multi-disciplinary open access archive for the deposit and dissemination of scientific research documents, whether they are published or not. The documents may come from teaching and research institutions in France or abroad, or from public or private research centers.
L'archive ouverte pluridisciplinaire HAL, est destinée au dépôt et à la diffusion de documents scientifiques de niveau recherche, publiés ou non, émanant des établissements d'enseignement et de recherche français ou étrangers, des laboratoires publics ou privés. 


\title{
POSSIBILITÉS D’APPLICATION DES GOUCHES MINCES MAGNÉTIQUES A LA DÉTEGTION DES GHAMPS MAGNÉTIQUES FAIBLES
}

\author{
Par B. WAKSMANN, \\ Section Physique des Couches Minces, C.N.R.S., Gedex I66, 38-Grenoble-Gare.
}

\begin{abstract}
Résumé. - Nous avons effectué une étude sur les possibilités d'application des couches minces ferromagnétiques comme élément sensible à la détection des champs magnétiques très faibles. Les couches minces ont été préparées par évaporation sous vide en présence d'un champ magnétique et possèdent donc une anisotropie induite uniaxiale dans leur plan dont l'axe est appelé axe de facile aimantation.

Le principe d'application, déjà connu $[2,3]$, repose sur la base suivante : un champ magnétique sinusoïdal $(100 \mathrm{kHz})$ d'amplitude grande devant le champ coercitif ou le champ d'anisotropie de la couche mince, excite l'aimantation de celle-ci le long de son axe facile ou de son axe difficile, la détection se fait par une bobine placée perpendiculairement au champ d'excitation. En présence d'un champ magnétique ambiant, la tension induite dans la bobine de détection indique la direction et la grandeur de la composante axiale de ce champ.

Nous avons été limités par suite des fluctuations basse fréquence du signal détecté à la mesure de $5 \times 10^{-6}$ Oe qui constitue actuellement le plus petit champ détectable par ce montage ; la bande passante était volontairement limitée à $5 \mathrm{~Hz}$, mais le montage permettrait de mesurer des champs de haute fréquence jusqu'à $50 \mathrm{kHz}$.

Nous décrirons le dispositif expérimental ainsi réalisé dont les avantages sont la faible consommation $(450 \mathrm{~mW})$ et le faible encombrement. Nous nous sommes attachés à diminuer au maximum la dérive et le bruit de l'électronique. Ainsi les fluctuations résiduelles qui limitent actuellement la mesure proviennent des couches utilisées. Parmi celles-ci, le rapport signal sur bruit optimum est obtenu pour les valeurs les plus faibles de la dispersion de l'axe facile et du champ d'anisotropie. Diverses structures magnétiques ont été étudiées, en particulier des films de FeNi polycristallins ou épitaxiaux, des films couplés.

Les fluctuations observées peuvent avoir deux origines, soit une propriété générale des ferromagnétiques (fluctuation thermique, traînage), soit des effets particuliers aux structures en couches minces (inhomogénéités, basculement incohérent).
\end{abstract}

Abstract. - We have made a study on the possible applications of thin ferromagnetic films, used as sensitive elements, to the detection of very small magnetic fields. The films, during their evaporation under vacuum, were submitted to a magnetic field, thus inducing a uniaxial anisotropy parallel to an axis in their plane, called the easy axis.

Their use as field detectors can be explained thus $[2,3]$ : the magnetization of the film, either along the easy or hard axis is submitted to a $100 \mathrm{kHz}$ sinusoidal magnetic field, large if compared to the coercitive or anisotropy fields; detection is made through a coil at right angle to the applied field. In the presence of an ambient field, the induced signal in the pick-up coil shows both the direction and intensity of the axial component of this field.

Low frequency fluctuations of the detected signal sets the threshold to $5 \times 10^{-6}$ Oe ; we have set the bandwith to $5 \mathrm{~Hz}$ but we can bring it up to $50 \mathrm{kHz}$.

The apparatus that we will describe has the advantages of requiring low operating power $(450 \mathrm{~mW})$ and small physical dimensions. Emphasis was to minimize noise and optimize stability; thus the limitating residual fluctuations are essentially those of the films themselves. For example a minimum Signal Noise Ratio is obtained for those with a minimum dispersion of the easy and anisotropy axis. Several structures were studied : polycristalline or epitaxial and coupled FeNi films.

The observed fluctuations may be due either to a general property of ferromagnetic materials (thermal fluctuation, magnetic aftereffect) or to properties inherent to thin films (inhomogeneities, non coherent rotation).

I. Introduction. - Nous avons effectué une étude sur les possibilités d'application des couches minces ferromagnétiques comme élément sensible pour la détection des champs magnétiques très faibles. Les couches minces étudiées ont été préparées par évaporation sous vide $\left(10^{-6} \mathrm{~mm}\right.$ mercure) en présence d'un champ magnétique continu qui leur confère une aniso- tropie induite uniaxiale contenue dans leur plan. L'axe d'anisotropie est appelé axe de facile aimantation. Ces couches minces présentent généralement dans leur direction facile un cycle d'hystérésis rectangulaire. La valeur du champ nécessaire pour renverser l'aimantation d'un sens à l'autre est appelée champ coercitif $H_{\mathrm{c}}$. La caractéristique suivant l'axe de difficile 
aimantation est linéaire avec saturation et peut aussi présenter parfois une hystérésis définissant un champ critique $H_{\text {ct }}$. Le champ nécessaire pour atteindre la saturation dans cette direction est appelé champ d'anisotropie $H_{\mathrm{K}}$ (fig. $\left.1 \mathrm{a}\right)$.

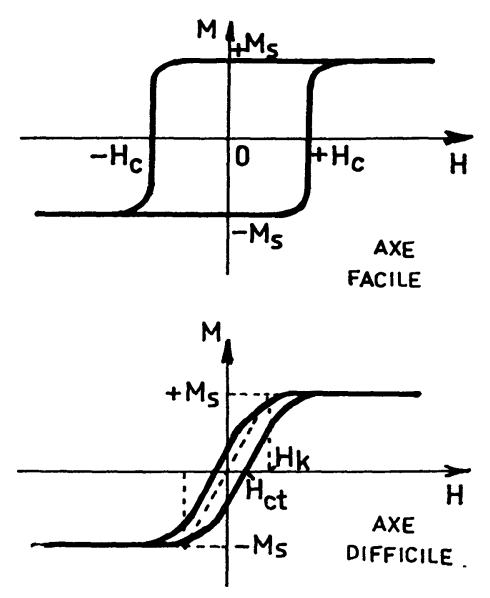

a

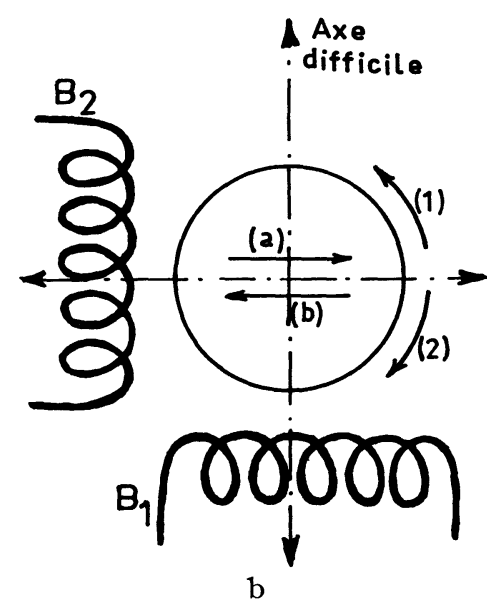

FIG. 1. - Cycles d'hystérésis et principe du capteur.

II. Principe de fonctionnement du magnétomètre. - Appliquons dans l'axe facile d'une couche mince un champ alternatif d'amplitude supérieure au champ coercitif $H_{\mathrm{c}}$ de la couche par l'intermédiaire d'un bobinage $\mathrm{B}_{1}$ ( fig. $1 \mathrm{~b}$ ); à chaque demi-période, l'aimantation va passer de la position (a) à la position (b), positions correspondant à la saturation de la couche mince dans la direction du champ appliqué.

Dans un modèle idéalisé de rotation uniquement cohérente de l'aimantation, et en l'absence de tout champ continu appliqué, il y aura, par raison de symétrie, autant de spins basculant dans le sens (1) que dans le sens (2), et la variation de flux résultante dans le bobinage $B_{2}$ sera nulle. Si maintenant on applique un petit champ continu dans l'axe difficile, l'aimantation basculera préférentiellement dans un sens déterminé par ce champ et un signal apparaîtra aux bornes de $\mathrm{B}_{2}$. En outre, nous remarquons que la phase de ce signal donnera le sens du champ appliqué.

Dans la pratique, le basculement de l'aimantation de sa position (a) à sa position (b) ne se fait pas aussi simplement et tout le fonctionnement du magnétomètre sera gouverné par le comportement réel de l'aimantation durant son renversement.
Il faut tenir compte, en réalité, du fait qu'il existe une dispersion de l'axe facile. Cette dispersion d'axe d'anisotropie est représentée par une fonction de distribution le plus souvent considérée comme gaussienne [1] (fig. 2 a) :

$$
f=f_{0} \mathrm{e}^{-\alpha^{2} / \alpha_{0}^{2}}
$$

où $f$ est donc la fonction de distribution par rapport à une direction moyenne (axe $o x$ ), $\alpha$ l'angle de la direction considérée avec $o x, f_{0}$ et $\alpha_{0}$ étant des constantes.
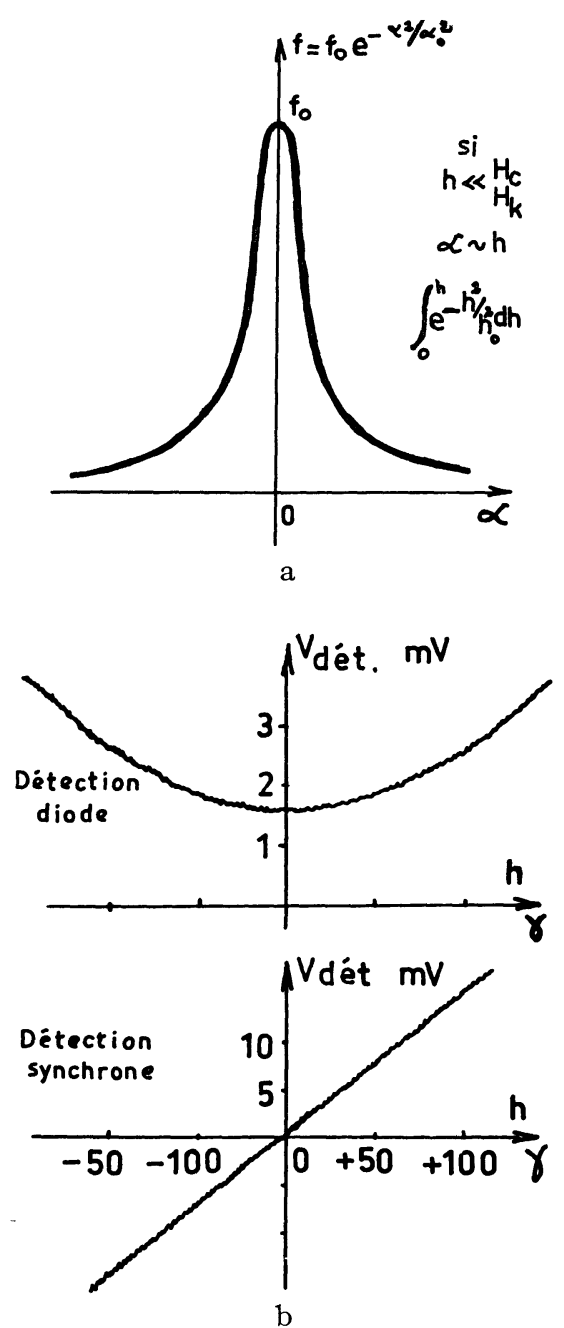

Fig. 2. - Dispersion d'axe facile et tensions détectées.

Pour de petits champs $h$ suivant l'axe difficile, donc de petits angles $\alpha$ :

$$
\sin \alpha \simeq \alpha \simeq \frac{h}{H_{w}}
$$

où $H_{w}$ est le champ critique de renversement; donc :

$$
f=f_{0} \mathrm{e}^{-h^{2} / h_{0}^{2}}
$$

où $h_{0}$ est une constante, et le signal qui apparaîtra donc au secondaire sera proportionnel à l'intégrale :

$$
\int_{0}^{h} \mathrm{e}^{-h^{2} / h_{0}^{2}} \mathrm{~d} h
$$

en prenant le développement en série au voisinage de $h=0$, le signal sera de la forme :

$$
S=K \frac{h}{h_{0}}\left(1-\frac{1}{3} \frac{h^{2}}{h_{0}^{2}}\right) .
$$


Il devrait être nul en champ nul. La sensibilité sera donnée par :

$$
\frac{\mathrm{d} S}{\mathrm{~d} h}=\frac{K}{h_{0}}\left(1-\frac{h^{2}}{h_{0}^{2}}\right)
$$

Il est à remarquer que la sensibilité est maximum pour $h=0$. Le magnétomètre sera prévu pour fonctionner toujours dans des champs très faibles (par exemple, en compensant le champ à mesurer par une troisième bobine) et la sensibilité sera constante et égale à $K / h_{0}$.

La figure $2 \mathrm{~b}$ est un enregistrement du signal total de sortie du capteur obtenu en détection diode en fonction du champ appliqué. La partie arrondie remarquée sur la courbe pratique s'explique par le fait que, pour $h=0$, le signal résiduel n'est pas nul et que la phase du signal varie rapidement : l'amplitude devenant de l'ordre du signal résiduel, la variation de tension efficace devient très faible. La détection de phase est alors nécessaire et nous l'avons réalisée sur un harmonique du signal de pompage. La figure $2 \mathrm{~b}$ montre que nous retrouvons une sensibilité constante dans les champs faibles.

Nous dénoterons $\alpha_{50}$ et $\alpha_{90}$ les valeurs de $\alpha$ correspondant à $50 \%$ ou $90 \%$ de l'aimantation tournant dans un sens déterminé.

Enfin, il faudrait tenir compte aussi en réalité du renversement par déplacement de parois, de la rotation incohérente de l'aimantation, effets qui peuvent aussi influencer fortement le fonctionnement $\mathrm{du}$ magnétomètre.

III. Dispositif expérimental. - Nous avons préféré au dispositif classique des magnétomètres à «fluxgate » le principe adopté par West et al. [2] et Castro et al. [3] qui nous semble mieux adapté aux couches minces.

En outre, en détectant perpendiculairement à l'excitation, nous pouvions envisager une étude plus large en ce sens que l'on peut soit exciter le long de l'axe facile et détecter sur l'axe difficile, soit le contraire, c'est-à-dire avoir un dispositif souple englobant les deux systèmes déjà mis en œuvre; ces deux fonctionnements devraient être différents, car l'excitation, selon l'un ou l'autre de ces deux axes, ne favorise pas le même type de renversement de l'aimantation.

Dans cette méthode, le signal apparaissant au bobinage de détection est un signal complexe composé de la fréquence d'excitation (fondamental) et de tous ses harmoniques. On ne détecte que sur un harmonique déterminé, un compromis étant fait entre le niveau de signal disponible et la sensibilité obtenue, une étude préliminaire ayant montré que cette dernière est d'autant plus élevée que l'ordre de l'harmonique est plus grand (de $H_{1}$ à $H_{20}$ ). On n'utilise ainsi que la fraction du signal correspondant à un basculement relativement rapide de l'aimantation.

Le dispositif expérimental finalement adopté est composé d'un ensemble électronique et du capteur proprement dit.

III.1. Le Gapteur. - Il est constitué par la couche mince placée dans un plateau en plexiglas permettant une orientation de celle-ci par rapport à deux bobines, l'une d'excitation, l'autre de détection. Ces bobines se glissent, axes perpendiculaires sur le support du plateau portant la couche.

L'ensemble est placé dans une boîte de centrage permettant de disposer le capteur au centre d'une chambre blindée. L'orientation de la couche par rapport aux bobinages peut être réalisée. Les deux bobines coulissent l'une sur l'autre, et par une légère retouche il est possible de régler leurs axes perpendiculaires, ceci est fait par annulation du couplage de mutuelle inductance entre elles.

III.2. L'ensemble Élegtronique. - Le schéma synoptique du dispositif est montré figure 3. La partie

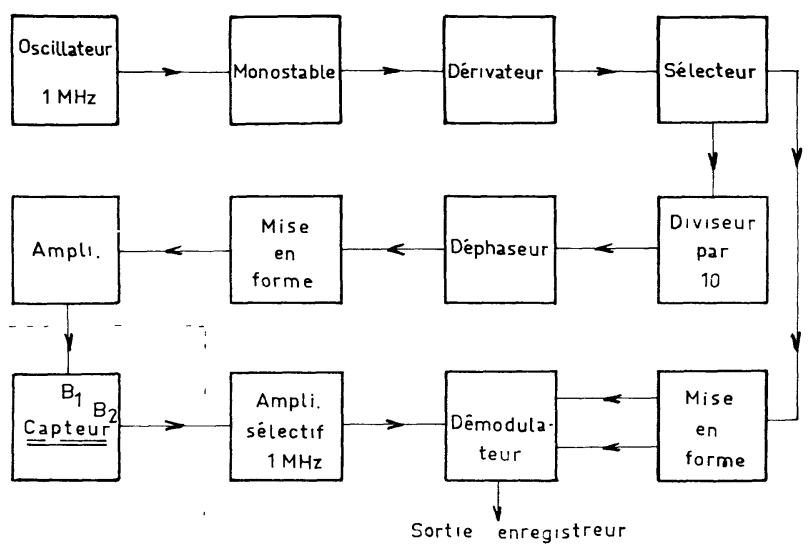

FIG. 3. - Schéma synoptique.

électronique est composée d'un oscillateur à quartz qui délivre un signal sinusoïdal de fréquence $1 \mathrm{MHz}$. Ce signal est envoyé dans un monostable, puis dérivé, et nous sélectionnons par diode les impulsions négatives. Ces impulsions déclenchent un monostable dont la sortie est envoyée :

a) D'une part, dans un diviseur par 10 (SM 92 Sylvania) donnant un signal carré à $100 \mathrm{kHz}$ et un signal de synchronisation pour oscilloscope. Le signal carré à $100 \mathrm{kHz}$ est envoyé dans deux étages à circuits intégrés (l'un permettant de faire varier la phase, l'autre étant un circuit de mise en forme). Le signal extrait est envoyé dans deux étages préamplificateurs, suivi d'un amplificateur de saturation et de mise en forme délivrant le signal sinusoïdal à $100 \mathrm{kHz}$ d'excitation de la couche;

b) D'autre part, dans un étage déphaseur délivrant des signaux carrés en oppositions de phase pour l'attaque d'un démodulateur à diodes.

Le signal extrait de la couche, composé de tous les harmoniques de la fréquence de pompage, est envoyé dans un amplificateur à circuits accordés à $1 \mathrm{MHz}$. Après cette amplification sélective, il est injecté dans le démodulateur à diodes.

La bande passante de la sortie détectée a été volontairement limitée à $5 \mathrm{~Hz}$, mais le montage permettrait de mesurer des champs de haute fréquence jusqu'à $50 \mathrm{kHz}$. Le niveau de bruit ainsi obtenu en sortie pour l'ensemble de l'électronique, et sans couche mince, est de $50 \mu \mathrm{V}$.

IV. Résultats. - Nous avons effectué avec ce dispositif expérimental l'étude de couches de propriétés variables :

a) Couches de $\mathrm{FeNi}$ polycristallines déposées sur divers supports, afin d'obtenir des caractéristiques particulières de cycles d'hystérésis dans l'axe facile et dans l'axe difficile. 


\begin{tabular}{|c|c|c|c|c|c|c|c|c|}
\hline Nos COUCHES & MATÉRIAUX & $\begin{array}{c}\text { ÉPAISSEUR } \\
(\AA) \\
-\end{array}$ & REMARQUES & $2 \alpha_{50}(\mathrm{Gr})$ & $H_{\mathbf{c}}(\mathrm{Oe})$ & $H_{\mathrm{K}}(\mathrm{Oe})$ & $H_{\text {et }}(\mathrm{Oe})$ & $\mathrm{B}(\gamma)$ \\
\hline FN 7a & FeNi sur SiO sur verre & $\begin{array}{l}10000 \mathrm{SiO} \\
2000 \mathrm{FeNi}\end{array}$ & $83 \% \mathrm{Ni}, 17 \% \mathrm{Fe}$ & 2 & 3,1 & 4,2 & 0,35 & 3,5 \\
\hline FN 7b & FeNi sur verre & 2000 & $n$ & 8 & 2,9 & 4,1 & 0,25 & 4 \\
\hline FN 5.2 & FeNi sur Cr sur verre & $\begin{array}{c}500 \mathrm{Cr} \\
1500 \mathrm{FeNi}\end{array}$ & $"$ & 6 & 3,5 & 3,1 & 1 & 4,5 \\
\hline FN 14.1 & FeNi sur verre O.P. & 1500 & ” & 2,5 & 2,3 & 2,9 & 0 & 1 \\
\hline FN 17.1 & $\begin{array}{c}\text { FeNi sur verre } \\
(\text { O.P. } \equiv \text { Optiquement Plan) }\end{array}$ & 6000 & $82 \% \mathrm{Ni}, 18 \% \mathrm{Fe}$ & 45 & 4,4 & 8,3 & 3 & 15 \\
\hline $\begin{array}{l}\text { FN.S.FN } 38.1 \\
\text { FN.S.FN } 38.2\end{array}$ & $\begin{array}{c}\text { FeNi sur SiO sur } \\
\text { FeNi sur verre O.P. }\end{array}$ & $\begin{array}{l}800 \mathrm{FeNi} \\
200 \mathrm{SiO} \\
800 \mathrm{FeNi}\end{array}$ & Couches triples & 1,5 & 0,95 & 4,3 & 0,25 & 20 \\
\hline M 50.1 & FeNi sur Ag sur mica & 1000 & & & 2,4 & 2,9 & 0,25 & 1 \\
\hline M 43.2 & $"$ & $"$ & Couches épitaxiales & & 2 & 2 & 0,35 & 1,2 \\
\hline FN 51.4 & $”$ & 1500 & $\sim 2000 \AA \mathrm{Ag}$ & 6 & & 2,4 & & 5 \\
\hline FN 63 & $"$ & $"$ & & 1 & & 2,3 & & 0,5 \\
\hline FNC 25.5 & FeNiCo sur verre O.P. & 1500 & $3 \%$ Co & 2,5 & 2,4 & 3,5 & 0 & 3 \\
\hline FNC 35.1 & $"$ & 1000 & Recuit $300^{\circ} \mathrm{C}$ & 2 & 2,2 & 2,7 & 0 & 1,5 \\
\hline FNC 35.2 & $"$ & 1500 & sous vide $7 \mathrm{~h}$ & 1 & 2,3 & 3,2 & 0 & 0,6 \\
\hline FNC 39.4 & $"$ & 1000 & $3 \%$ Co & 3,5 & 2,9 & 3,6 & 0,7 & 5 \\
\hline FNC 39.5 & $"$ & 5000 & $"$ & 3 & 1,7 & 2,75 & 0,12 & 0,8 \\
\hline FNC 39.6 & $"$ & 1500 & $"$ & 1,5 & 2,6 & 3,2 & 0,12 & 1 \\
\hline FNC 40.5 & $"$ & 2500 & $6 \% \mathrm{Co}$ & 0,5 & 3 & 8 & 0,9 & 4 \\
\hline
\end{tabular}

FIG. 4. - Caractéristiques de quelques couches.

b) Couches de FeNiCo avec faibles pourcentages de cobalt $(3 \%$ et $6 \%)$ de façon à ne pas trop accroître les champs coercitifs. Ces couches présentent généralement une dispersion plus faible.

c) Couches triples FeNi-SiO-FeNi, mais possédant un cycle unique.

d) Couches épitaxiales de FeNi déposées sur argent lui-même déposé sur mica, qui possèdent à la fois des dispersions faibles, des champs d'anisotropie faibles, et dans lesquelles on peut espérer obtenir une homogénéité des propriétés plus grande que dans les couches polycristallines.

e) Couches de dimensions diverses permettant d'évaluer les effets des formes, en particulier les effets de champ démagnétisant. Le tableau de la figure 4 donne les caractéristiques de quelques couches obtenues.

Nous avons mesuré le rapport signal sur bruit global du magnétomètre équipé avec chacune de ces diverses couches.

De ce point de vue, certaines couches s'avèrent comme franchement mauvaises, ne permettant que la mesure de champs de l'ordre de $1000 \gamma$ ou de $10000 \gamma$. Par contre, de nombreuses couches donnent un bruit global équivalent à des champs compris entre 1 et $10 \gamma$, la limite optimale étant de $0,5 \gamma$. Dans ce dernier cas, le niveau de fluctuation à la sortie du montage est de l'ordre de $50 \mu \mathrm{V}$ sans couche et de $500 \mu \mathrm{V}$ à $700 \mu \mathrm{V}$ avec couche. Nous avons donc été amenés à considérer que l'origine du bruit était certainement à rechercher dans le film magnétique lui-même. Toutefois, le signal d'excitation étant de grande amplitude, seuls le bruit et les fluctuations de très faibles amplitudes de ce signal n'ont pu être mesurés, et il subsiste un doute quant à l'effet que pourraient avoir de telles fluctuations, même de très faible amplitude, sur le signal détecté.

Nous avons essayé de comparer le rapport bruit/signal obtenu avec chaque échantillon, et les divers paramètres de cet échantillon, c'est-à-dire $H_{\mathrm{c}}, H_{\mathrm{K}}$, $H_{\text {ct }}$ et $\alpha_{90}$. Aucune loi générale ne lie le rapport $\mathrm{B} / \mathrm{S}$ simplement à l'un de ces paramètres.

Toutefois, pour des échantillons, de même matériau, de mêmes épaisseurs, préparés dans les mêmes conditions, et donnant les meilleurs résultats $(\mathrm{B} / \mathrm{S}$ minimum), la figure 5 , donnant le bruit en fonction

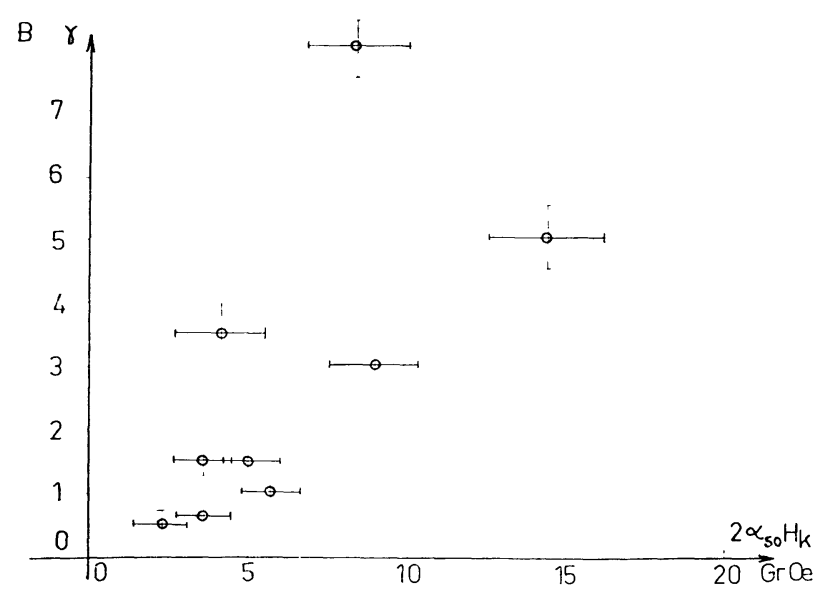

FIG. 5. - B en fonction du produit $2 \alpha_{50} H_{\mathbf{K}}$. 
du produit $2 \alpha_{50} H_{\mathrm{K}}$, montre, malgré la dispersion des résultats, que le rapport bruit/signal croît avec ce produit.

Malheureusement, ce produit ne peut être encore fortement diminué, tout du moins avec les matériaux et les techniques de préparation dont nous disposons.

Si l'excitation est faite dans l'axe difficile et la détection dans l'axe facile de la couche, pour les échantillons où le rapport $\mathrm{B} / \mathrm{S}$ est faible, ce dernier est identique à la valeur relevée dans le mode d'excitation précédent. Par contre, pour des rapports B/S élevés, il peut être, selon les couches, nettement plus faible ou nettement plus fort dans ce mode d'excitation.

Nous avons effectué quelques mesures en fonction de la température dans la gamme $-60^{\circ} \mathrm{C}$ à $+40^{\circ} \mathrm{G}$ et, en dernier lieu, à $-193^{\circ} \mathrm{C}$ en plaçant le capteur directement dans l'azote liquide.

Dans toute cette gamme, la dérive est importante, mais lorsque la température s'est stabilisée, nous n'avons observé aucune différence, ni sur la sensibilité, ni sur le bruit résiduel.

Le niveau de bruit observé sur l'ensemble des structures étudiées montre donc que dans les meilleures conditions et pour des échantillons divers, nous obtenons au mieux $0,5 \gamma$. Cette limite nous conduit à considérer les diverses origines possibles de ce bruit.

- Bruit ferromagnétique analogue aux fluctuations existant dans les matériaux massifs et étudié par L. Néel, sous le titre général de Traînage magnétique [4 à 7].

- Fluctuations d'origine thermique (sur les domaines ou sur les spins). Dans ce cas, l'effet de la température doit être mesurable mais aucune expérience n'a été faite dans ce sens puisqu'il faudrait étudier la limite du spectre de bruit en fonction de la température et non le spectre aux fréquences moyennes comme nous l'avons fait.
- Phénomène de rotation incohérente de l'aimantation (propre aux couches minces monodomaines).

- Inhomogénéités des propriétés magnétiques des films (composition de l'alliage, dispersion de champ d'anisotropie ou défauts structuraux). Ces inhomogénéités peuvent avoir une grande importance comme semblent le montrer nos mesures faites en fonction de l'amplitude du champ d'excitation de la couche [8].

V. Conclusions. - Nous avons réalisé un dispositit expérimental permettant la détection des champs magnétiques faibles et utilisant des couches minces ferromagnétiques comme élément sensible. Les avantages de ce dispositif sont la faible consommation $(450 \mathrm{~mW})$ et le faible encombrement.

Sa résolution, de $0,5 \gamma$, semble difficile à améliorer actuellement par la recherche de couches présentant des caractéristiques meilleures. Par contre, la connaissance de l'origine du bruit limitant la mesure et dont l'étude reste à faire, pourra peut-être permettre l'amélioration de ses caractéristiques de détection. En particulier, deux études peuvent être envisagées :

- L'une serait l'étude du spectre de bruit Barkhausen, effectuée de la même façon que les études entreprises dans les matériaux massifs, en basculement très lent, cycle par cycle [9 à 20].

- L'autre serait l'étude du fonctionnement du magnétomètre en utilisant uniquement le basculement cohérent de l'aimantation, ce qui exigerait des fréquences d'excitations plus élevées, soit même une excitation par impulsions.

Remerciements. - Nous tenons à remercier très vivement le Gentre National d'Études Spatiales qui a subventionné cette étude (Convention 68 CNES 207), M. J. C. Bruyère, qui a constamment suivi son développement, M. A. Plante, qui a réalisé la partie électronique, et Mlle C. Bonvarlet, qui a préparé les couches minces.

\section{BIBLIOGRAPHIE}

[1] Koor (C. F.), Theory of Magnetometer, Rapport Société Lockheed, non publié

[2] West (F. G.), Odom (W. J.), Rice (J. A.) et PEnN (T. C.), J. Appl. Phys., 1963, 34, 1163.

[3] Castro (P. S.) et Stucki (F. F.), Brevet d'Invention no 1085682, 1966, de Société Lockheed Aircraft Corporation.

[4] Níé (L.), J. Physique Rad., 1950, 11, 49.

[5] NÁÉ (L.), J. Physique Rad., 1951, 12, 339.

[6] NéÉ (L.), J. Physique Rad., 1952, 13, 249.

[7] BARbier (J. C.), Thèse de Docteur ès Sciences, 1953.

[8] Waksmann (B.), Bruyàre (J. C.), Plante (A.) et BONVARLET (C.), Rapport Contrat 68 CNES 207, non publié.

[9] Mazzetiti (P.) et Montalenti (G.), J. Appl. Phys., 1963, 34, 3223.

[10] MazzeTti (P.) et Montalenti (G.), Conf. on Mag., Nottingham, 1964, 701.
[11] Bitrtel (H.) et Westerboer (I.), Ann. Physik, 1959, 4, 203.

[12] Birter (H.), Cours d'été OTAN/Marine Nationale "Traitement du signal ", CEPHAG 489-516, Grenoble, 1964.

[13] Stierstadt (K.) et Pfreinger (E.), Z. Physik, 1964, 179, 182.

[14] Stierstadt (K.) et Geile (H. J.), Z. Physik, 1964, $180,66$.

[15] STORM (I..), HFiden (C.) et Grosse-Nobis (W.), I.E.E.E., Trans. Mag., 1966, 2, 434.

[16] Lambeck (M.), J. Appl. Phys., 1968, 39, 741.

[17] Ferro (A.), Mazzetuti (P.) et Montal finti (G.), Il Nuovo Cimento, 1968, 56, 111.

[18] Aroues (P. Y.), J. Physique, 1968, 29, 369.

[19] Zentko (A.) et Hajko (V.), Czech. J. Phys., 1968, B 18, 1026.

[20] Rudyak (V. M.) et Kharitonov (Yu. N.). Sov. Phys. Doklady, 1968, 13, 38. 